\title{
RAZÓN: ¿CRITERIO O CULTURA? PARA LA DEFINICIÓN DE LO RACIONAL
}

FRIEDRICH KAMBARTEL

UNIVERSIDAd dE CONSTANza

Trazar líneas fronterizas entre lo racional y lo irracional es una actividad favorita de los filósofos. Algunos de nosotros nos ocupamos o nos hemos ocupado profundamente en esas arduas tareas. $Y$ también dentro de una perspectiva histórico-filosófica, una investigación sobre lo que cabria llamar "racionalidad" o "razón" nos conduce a campos en extremo conocidos. (Cuando se utiliza lo irracional en un sentido peyorativo, normalmente tenemos que distinguir entre lo racional, lo no-racional y lo irracional.)

Algunas veces hasta podríamos tener la impresión de que las escuelas o los movimientos filosóficos se diferencian unos de otros en última instancia precisamente por sus conceptos de racionalidad. ¿Y acaso no es también la tarea constitutiva o incluso la misión de la filosofía aclarar y fomentar la idea de la razón? En el campo de la práctica, la presente perspectiva nos conduce en particular a controversias en las cuales se contraponen las definiciones de racionalidad técnicas o extraídas de intereses personales a los conceptos morales de la razón, en el sentido de una comprensión de la moralidad que no entraña únicamente una "racionalidad de fines": "entendimiento" ["Verstand"] versus "razón" ["Vernunft"], como lo ha expresado el lenguaje filosófico alemán clásico.

En esta ponencia no tengo la intención de lidiar con las palabras "razón" o "racionalidad". Ustedes tendrán que descubrir por sí mismos que esto no es necesario para nuestra presente investigación, sobre todo porque en este momento no me adentraré en ulteriores tareas de delimitación (sobre la comprensión "correcta" de la razón). Tampoco construiré, propondré o justificaré nuevas definiciones de racionalidad, ni me ocuparé de las viejas "precisiones" para modificarlas o atacarlas directamente. Lo que en las reflexiones que siguen me incumbe esencialmente es la forma que toman en muchos casos (quizá en la mayoría de los casos) las disputas (filosóficas y científicas) sobre el concepto de razón. Por ello, 
mi objeto es, sobre todo, la razón práctica, 'aunque en lo que sigue no se ponga de manifiesto continuamente esta restricción de mis propósitos. Mucho de lo que voy a decir es igualmente válido para el uso teórico de la razón, que, por lò demás, está incluido en el uso práctico.

Normalmente utilizamos la palabra "racional" para expresar una valoración. Calificamos argumentos, nuestra conducta, o a una persona, como racionales, decimos que muestran razón, etc. Como evidentemente no podemos en estos casos simplemente "percibir" la racionalidad, surge la idea de que aplicamos una especie de norma [Maßstab] o criterio [Standard]: una norma contenida en la gramática de las palabras "racional" y "razón". Así pues, las controversias ifilosóficas sobre el concepto de racionalidad toman de un modo aparentemente forzoso la forma de contiendas sobre la norma correcta de lo racional. Cuando además nos fijamos en la sola palabra "racional", con su extendidísimo uso, resulta patente que también nosotros tenemos que buscar una medida o principio general de la razón (entretejido en todo este uso), un principio que sirva de base en última instancia a todos los juicios pertinentes. Si los conceptos de razón que entran en conflicto en la filosofía, pero también en otras disciplinas como la economía y la teoría de la decisión, se refirieran a diferentes tipos de situaciones e intenciones, las controversias se resolverían a fin de cuentas con sólo ponerse de acuerdo sobre las restricciones gramaticales (conceptuales) necesarias.

Normalmente, sin embargo, esto no es posible; normalmente nos enfrentamos en estas controversias con principios o criterios de la razón o la racionalidad (práctica) que aspiran a poseer una relevancia general y que por lo mismo no pueden hacerse fácilmente compatibles al vincularlos con diferentes contextos. Permítaseme recordar dos ejemplos bien conocidos. Dado que no prestaré atención a los detalles, hablaré, más que de ejemplos; de grupos de ejemplos.

Llamaré representación de un principio de razón a la idea de una norma universal (es decir, aquí, de aplicación general) de juicio, tal como se ilustra en ambos ejemplos (grupos de ejemplos). El primer principio de esa índole que quièro discutir es el que se llama, con una caracterización muy usual, principio del interés personal racional. La concepción o, quizá mejor, la sugestión antropológica que rige para este principio (para los principios de este grupo), es aproximadamente la siguiente:

Está ante todo supuesto un concepto que ya es accesible en el lenguaje común de lo que significa para una persona tener intereses, intereses que, como intereses de esta persona, calificamos, bajo diferentes perspectivas, como subjetivos, individuales o privados. Tener 
intereses y seguirlos en nuestras acciones se convierte entonces en un rasgo universal fundamental de la situación vital humana. Más aún, parece que fácilmente podemos ver que existen intereses con estructuras determinadas, las cuales tienen siempre la misma forma, aunque su contenido pueda ser diferente de una persona a otra.

En primer lugar, parece que nuestros intereses dependen unos de otros, en el sentido de que la prosecución o la realización de algunos intereses determinados forma parte de las condiciones suficientes, o a veces también necesarias, para la satisfacción de otros intereses. Por otro lado, existen relaciones de preferencia entre los intereses, relaciones que acaso pueden comprenderse con base en las dependencias arriba mencionadas, pero que más frecuentemente se exhiben como reacciones o actitudes inmediatas ante la comparación de intereses. Las relaciones de dependencia y preferencia, y tal vez otras relaciones, hacen que nuestros intereses aparezcan jerárquicamente ordenados. $Y$ este ordenamiento de nuestros intereses nos da finalmente - así parece - la posibilidad de alcanzar la mejor elección posible entre las posibilidades de acción disponibles en cada caso, y en este sentido, alcanzar las estrategias racionales de acción. La forma particular de este "cálculo" de nuestros intereses y acciones, $o$, en otras versiones, de nuestras preferencias, estimaciones de utilidad, etc., es algo muy discutido; discutido no sólo en filosofía, sino también en economía y en la teoría de la decisión racional, donde encontramos toda una serie de los llamados modelos exactos para el armazón (conceptual) y la aplicación de un principio de interés personal racional.

Si alguien quisiera sostener (aludiendo a nosotros) que el cuadro trazado de ponderación racional de los intereses personales es en muchos casos fácilmente imaginables una descripción adecuada, nadie lo contradiría. Tal vez habría que agregar, delimitando las cosas, que el cuadro sugiere que siempre tenemos que mirar y calcular la totalidad de nuestros intereses para obtener una orientación racional. Aunque pueda haber situaciones que aconsejen esta forma global de ponderación, éste es un caso extremo para el que es difícil imaginar un posible cálculo detallado. Sin embargo, en casos particulares y para fines determinados, el cuadro puede constituir una caracterización adecuada. Las dificultades radican en su uso como un principio (universal), que, en cuanto una comprensión [Verständnis] de aplicación general, tendría que extenderse, por ejemplo, también a las discusiones morales. No se le permitiría entonces a la razón moral mantenerse sobre sus propios pies.

La tradición ética tomó en particular dos caminos cuando se trataba de poner la argumentación moral bajo el principio del interés 
personal racional. La primera propuesta le atribuye al buen samaritano èl interés (subjetivo) en una situación general en la que también él pueda esperar la ayuda de sus prójimos sin una recompensa inmediata. En sus llamadas orientaciones "morales" el buen samaritano calcula por tanto sus intereses sólo en una perspectiva suficientemente amplia. Podríamos denominar a esta versión el núcleo de las mejores versiones de la posición utilitarista de la moralidad.

El segundo camino para una reconciliación entre el interés personal y la moralidad nos lleva a descripciones de situaciones como ésta: entre nuestros intereses esenciales o básicos se encuentra, para algunos de nosotros o para todos, un grupo especial: los intereses morales, entendidos como los intereses de experimentar determinadas sensaciones positivas (agradables, laudables) que acompañan la acción moral. El buen, samaritano disfruta de los imponentes sentimientos de simpatía que acompañan a las situaciones llamadas de ayuda "altruista"; y por medio de una evaluación correcta, aunque tal vez para él inconsciente, de sus intereses morales (privados), puede luego procurarse situaciones de ofrecimiento de ayuda aparentemente desinteresada, o por lo menos tomar las oportunidades que se le ofrecen, como lo hizo el buen samaritano de Lucas 10.

Espero que esto sea suficiente para recordar cómo la representación y el cálculo del interés personal racionalmente entendido adquieren la posición de un principio, de una norma de racionalidad comprehensiva; cómo funciona, en este caso, esta tendencia principal de los conceptos filosóficos de racionalidad.

Para la situación actual de la discusión, es acaso más ilustrativo el segundo principio de la razón, del cual me ocuparé ahora y al que llamaré principio del discurso racional. Así como el principio del interés personal racional, el del discurso racional es un principio formal de racionalidad. En el caso de principios formales de razón, la acreditación de la pretensión de racionalidad se apoya en el camino que nos̀ conduce a la justificación de una orientación, y no en una estimación valorativa directa de resultados determinados. Por lo demás "el camino" puede significar aquí muchas cosas, por ejemplo una manera determinada de la reflexión, la forma de la situación reflexiva, reglas de la fundamentación (justificación) racional o, por último, las actitudes de quien interviene en la reflexión. Pero mientras que el interés personal racional se refiere a las formas de un cálculo de intereses individual, en la base del principio del discurso se encuentra la idea de que las pretensiones de racionalidad están vinculadas a condiciones y actitudes que determinan la vía hacia una coincidencia de nuestros juicios, hacia un consenso que hemos 
alcanzado o que, de acuerdo con nuestro mejor saber y entender, podríamos alcanzar.

Ello significa lo siguiente: ante todo, en un primer sentido, las palabras "racional" y "razón" sirven aquí para caracterizar una forma (formas determinadas) de situaciones comunes de reflexión y de juicio. Después, en un segundo paso, se caracteriza como racional una coincidencia alcanzada, precisamente cuando es el resultado de una discusión racionalmente configurada. Finalmente, en tercer lugar, una orientación, por ejemplo una verificación o una regla, cuenta como "racional" o como "racionalmente fundada" precisamente cuando podemos fundar convincentemente su valor en un consenso racional.

Si no me equivoco, esto corresponde grosso modo por ejemplo al proceder de Habermas, en las "Notizen zu einem Begründungsprogramm" de la "Diskursethik", contenidas en el volumen Moralbewußtsein und kommunikatives Handeln, aparecido en 1983, pero también corresponde a mis propias propuestas de explicación anteriores, que pueden consultarse en un tratado de 1974: Moralisches Argumentieren.

Ahora bien, existen numerosos intentos de reconstruir adecuadamente un principio discursivo de razón. Las condiciones consideradas como ingredientes de este tipo de análisis son, por ejemplo:

-la veracidad de los participantes: que presenten sus orientacioneş $\mathrm{y}$ argumentos seriamente $\mathrm{y}$ con mutua transparencia;

-la ausencia de sanciones (externas) (sólo la fuerza del mejor argumento debe determinar el asentimiento o el disentimiento);

-la simetría de las posiciones en la situación discursiva: que las reglas o las condiciones contextuales no le den una posición privilegiada a ninguno de los participantes.

Además de estas características, encontramos condiciones que buscan garantizar que el discurso racional atienda a todos los intereses relevantes ("a todos los afectados"). Tales condiciones, como la franqueza y la publicidad de las argumentaciones, son aparentemente indispensables si queremos relacionar el discurso racional con una perspectiva moral y distinguirlo de la racionalidad (y también solidaridad) interna de una banda de ladrones. Es obvio que los que de hecho participan en un discusión no serán normalmente idénticos que los afectados por sus objetos y sus resultados. Por eso el egoísmo grupal siempre es una posibilidad y debe ser, al parecer, excluido explícitamente para un análisis correcto y suficientemente preciso de la racionalidad. 
Como en el caso del interés personal racional, concederemos que las descripciones que acabamos de mencionar, cada una por sí misma, son bien observadas si las mantenemos contra conocidos usos de (y apelaciones a) "razón" o "racionalidad", en particular en situaciones en las que esas caracterizaciones no se cumplen. La cultura de la razón se desarrolla y se mueve en gran medida en.y con las formas imparciales del reflexionar, el juzgar y el resolver.- Y vuelven a presentarse disputas filosóficas bien conocidas al intentar agrupar las condiciones del discurso racional de tal forma que lleguemos a una definición de racionalidad, definición que sirva como un criterio general, como lo que llamo aquí un principio de razón, o por lo menos como un principio de razón para el dominio de las discusiones prácticas.

Tampoco aquí quisiera entrar en detalles, sino solamente decir, sin ninguna seguridad definitiva, que los esfuerzos de Apel, Habermas, pero también acaso los de Hare y Rawls, así como finalmente los de Lorenzen, Schwemmer o los de mis propios pensamientos anteriores, y tal vez en general los del kantismo como filosofía práctica, muestran a mi entender una tendencia común y de ninguna manera comprensible de suyo. Pero también las variedades del utilitarismo y de la economía del bienestar público, están afectadas precisamente por esta tendencia.

¿Acaso existe otra visión de la situación en la que, gramaticalmente, nos las habemos con las pretensiones de razón? (Al margen: mi continuo uso de la palabra "gramatical" puede entenderse como una referencia a Wittgenstein, quien fue acaso el primero que le dedicó suficiente atención a un planteamiento del problema del tipo del que me ocupo aquí. Naturalmente, en la mayoría de los casos la palabra "gramatical" podría sustituirse por las palabras "conceptual" o "semántico".)- Si por el momento dejamos de lado la idea de un principio general de razón, entonces nos quedamos con un conjunto de condiciones o criterios de racionalidad gramaticales, a algunos de los cuales estaríamos tentados a llamar "técnicos", y a otros, "morales". Algunos de ellos tienen la forma de reglas, otros se interpretan como una meta o una perspectiva, y otros más como una actitud o capacidad. Y estas "formas" pueden relacionarse luego con una multiplicidad de lo que podríamos llamar puntos de vista de contenido, como por ejemplo los puntos de vista de la simetría, la igualdad, el reconocimiento mutuo como persona (moral), la generalidad (de la formulación), la universalidad (de la aplicación), la justicia; o del tipo de la veracidad, el realismo y la verdad, pero también del tipo del interés personal calculado y la racionalidad técnica, $y$, finalmente, de la sabiduría y de la forma 
correcta (buena) de la vida individual o de la comprensión de la vida.

Pero ¿acaso no son todos estos aspectos (formales y materiales) de la razón meras facetas de uno y el mismo fenómeno, al que llamamos racionalidad?- Podemos hablar de esta manera, sólo con que no consideremos lo que acabo de llamar "aspectos" o "facetas" como notas de una definición de racionalidad, o las reduzcamos a implicaciones y construcciones semánticas que tengan como fundamento una definición semejante.- La búsqueda de construcciones lógicas - semánticas en las cuales se pueda apoyar un principio general de la razón, ha de ser vana. Y esto - la inutilidad del esfuerzo- vale también para las controversias filosóficas o científicas que se derivan de semejantes perspectivas. Aquí podría imponerse por tanto la despedida de una representación teórica, una despedida que quisiera darles a conocer.

Si la razón nos dirige, ¿seguimos entonces el (único) principio - criterio general de razón, una serie de reglas acaso? ¿O no deberíamos mejor decir que, como seres racionales cultos, somos capaces de juzgar con una gran variedad de criterios de valoración diferentes aunque relacionados unos con otros de múltiples maneras; lo cual quiere decir, en particular, que nos corresponde y nos es posible evaluar y comprender su adecuación y su correcta relación respecto de la situación de que en cada caso se trata? La cuestión de la adecuación, que aquí es esencial, no nos conduce de nuevo a un criterio general, sólo que "más elevado". Esto es, no existe ningún estándar general de adecuación (una marca) que pudiera luego aplicarse a todas las situaciones pensables. Por el contrario, hemos de decir que utilizamos y mostramos nuestra razón sólo en la medida en que conocemos (conocemos prácticamente) las normas [Maßstäbe] (de diferente especie) de adecuación a las situaciones y sus relaciones significativas respectivas, sin apoyarnos en criterios de juicio de un siguiente nivel, que estarían a nuestra disposición por todos lados.- Si quisiéramos emplear aquí expresiones de la filosofía kantiana, diríamos: la "Vernunft" (razón) no puede prescindir del "Urteilskraft" (juicio) ni de la experiencia vital que se halla en la base de éste. $Y$ decir esto no es una observación empírica, sino gramatical.

Si intentamos ahora modificar en este sentido la comprensión filosófica de la razón, necesitamos una palabra que pueda servir como contrapunto de palabras como "principio", "criterio" o "definición". Debe ser una palabra que se refiera a una gran variedad de prácticas y normas cuya coherencia no se consiga ni garantice mediante líneas de demarcación del tipo de una definición. Considero que la palabra 
"cultura" es una buena propuesta.- Esta palabra es indicada para nuestros propósitos en muchos respectos. Permítaseme referirme a algunos de ellos.

Ante todo, normalmente entendemos por cultura una práctica común y sus orientaciones, un fenómeno social. Y tampoco el compromiso que nos impone la razón es puramente autoinducido, ni se apoya únicamente en las decisiones privadas del individuo.

En segundo lugar, por lo común, la forma y el contenido de una cultura no se aprenden a través de una caracterización general. Si queremos tener un juicio competente sobre las actividades, distinciones, valoraciones y modos de resolver problemas que se desarrollan en y con una cultura (en nuestro caso, sobre lo que es racional), tenemos normalmente que alcanzar el conocimiento correspondiente conociendo y familiarizándonos con cada uno de los "componentes" de la cultura, "uno por uno", por así decirlo (y "paso a paso").Además, aquí una caracterización se alcanza más bien de modo ilustrativo, mediante "ilustración" [Erläuterung], en el sèntido fregeano, y no mediante "explicaciones" [Erklärungen] (definitorias). Más aún, las meras ilustraciones "externas" no nos dan en general la posibilidad de movernos prácticamente en una cultura, esto es, todavía no hacen de nuestra vida una parte de esta cultura. Aquí más bien se nos indica que lo necesario se nos enseña, que participamos prácticamente (no sólo en el plano de la descripción), que experimentamos; y en ello dependemos de otros (de la tradición respectiva) y también (dependemos) de las vicisitudes de la vida, que le dan a nuestra vida la variedad necesaria.

Debería agregar que la cultura de la razón es diferente de las culturas que determinan una práctica particular, un componente “local”, por así decirlo, en nuestra vida; la diferencia radica en que la razón, especialmente por sus conexiones gramaticales con el reflexionar, el argumentar, el orientarse, puede ser significativa en todos los dominios (de nuestra vida): en este sentido, el entendimiento y la práctica de la razón pertenecen a la forma de nuestra vida, es decir, a nuestra vida cuando la vemos como "un todo".

Nos encontramos, por lo demás, en una situación similar si buscamos principios definitorios de la libertad o de la moralidad. En vez de ello, aquí también habría que aconsejar poner la mirada en la red gramatical de una cultura (moral).

No nacemos como seres racionales, aunque, por nacimiento, estamos más o menos provistos para esta difícil empresa - hacia la razón nos formamos, mediante la formación se nos conduce a ella; y después nosotros mismos podemos contribuir a ella.

Imaginemos que falle el proceso de formación hacia la razón, o que 
se pierda la tradición que lleva consigo a la vida racional. ¿Podrían entonces (en su lugar) ayudarnos las definiciones y las delimitaciones teóricas, podrían ser acaso el fundamento gramatical para una renovación?

Tal vez dos comparaciones semánticas puedan arrojar más luz sobre la situación.

Representémonos lo racional en comparación con la solución adecuada de un problema - adecuada con respecto a la situación dada, a las circunstancias relevantes. De hecho, en muchos casos lo racional y lo adecuado van de la mano. Sin embargo, es manifiesto que sería totalmente absurda la idea de que tuviéramos que tener a la mano una norma general para soluciones adecuadas, una norma para situaciones-problema cualesquiera. La gramática de la palabra "adecuado" no es menos extensa y variada que la de la palabra "problema". Tenemos que saber primero de qué tipo de problema se trata, antes de que podamos señalar como tales las vías apropiadas para manejarlo; y esto, supuesto que dispongamos del juicio necesario.

También aquí podemos ver explicaciones del tipo "Esto no es correcto porque...", y luego darnos cuenta de que aquí la continuación, tras el "porque", es totalmente diferente y no es del mismo tipo de las que utiliza una definición (general) de "correcto".

Por otra parte, puede ser útil comparar el caso de una cultura con el de un juego: conocer un juego significa conocer una práctica particular y sus reglas. (Las reglas, por lo demás, no son esenciales para todos los juegos: pensemos en un juego en que algo tenga que representarse como en un teatro, acaso una situación o personajes determinados, y donde para empezar nada esté determinado y todo quede a la fantasía y a la intuición. Los niños pueden jugar de esta manera y, sin embargo, no seguir reglas en sentido restringido.)

Para nuestro propósito es útil pensar en un juego que se siga esencialmente por reglas definidas. 'Tomemos el ejemplo del ajedrez. Sería absurdo pensar que las reglas del ajedrez son "definibles" por el hecho de que satisfacen rasgos particulares, un "criterio" de lo ajedrecístico. Antes de conocer individualmente cada una de las reglas del ajedrez no hay nada (que sea común a ellas) que pueda conocerse acerca de las reglas. La pregunta: “ ¿Cómo sabes tú que ésta es una regla del ajedrez?" sería (aquí) un total absurdo, por lo menos en las situaciones en las que podemos pensar en una frase semejante.

Algo similar sucede -quisiera decir-con la información: es una justificación o una argumentación racional, porque obedece a la razón. Pues "la razón" no es un criterio que representara aquí una 
especie de control u ordenamiento para la disposición, ni siquiera cuando establecemos reglas del discurso o de la discusión en lugar (de la razón).

La perspectiva gramatical que he considerado guarda relación con una forma correcta de entender lo que a menudo se llama "el argumento trascendental" (en algunos casos se habla también de "an'álisis presuposicional" o de "contradicciones realizativas"): el argumento trascendental pone a la vista del destinatario lo que él hace, en comparación con lo que en su intención gramatical dice sobre lo que hace. La práctica del destinatario sería absolutamente imposible, en la forma de entenderla que (en la práctica) sirve incontestablemente de guía, si el destinatario, en sus afirmaciones acerca de la gramática de su práctica, estuviera en lo correcto. Si con nuestras formulaciones no hemos puesto en entredicho la fórmula kantiana de "condiciones de posibilidad", entonces quizá sería necesario expresar esto de manera menos ambigua: la gramática efectiva de la práctica del destinatario se opone a la descripción que éste hace de ella, y por eso el llamado argumento trascendental debe hacer referencia a él .

Desde luego, el destinatario puede modificar su práctica particular presente, para evadir la versión tu-quoque dél argumento trascendental. Pero lo que en circunstancias normales no puede hacer es cambiar la acción y la comprensión comunes, pues éstas están incorporadas en el contexto general de la vida, en la totalidad (la "forma") de nuestra (y también de su) vida. Una versión fuerte del argumento trascendental puede por ello referirse, y se referirá, al hecho de que el destinatario comparte con nosotros una gramática común, la "cultura" de nuestras vidas. Es obvio que el que esta gramática adquiera la forma de un principio (de aplicación general) no es de ninguna manera algo esencial para el argumento (trascendental).

Donde no existe ninguna cultura gramatical de la razón (donde la palabra "razón" y sus vecinos lingüísticos reciben un significado transsubjetivo), falta la instancia a la que deben remitirse los análisis filosóficos (de acuerdo con la índole del argumento trascendental). Si no podemos apelar a una cultura gramatical general de la razón, las indicaciones gramaticales para la comprensión de las pretensiones de razón siempre permanecerán en la cercanía de las meras decisiones (del decisionismo). Los filósofos y sus discípulos deben conocer la vida, la gramática de la vida que les es común, y poder remitirse a ella. La pluralidad o variedad gramatical que determina una cultura de razón no debe confundirse con el pluralismo: 
el pluralismo. de las opiniones en conflicto sobre (las concepciones de) la gramática de la racionalidad.

Sin embargo, es concebible que lleguemos a una situación en la que surja un conflicto entre nuestros análisis gramaticales y el desarrollo cultural. Es muy posible que una cultura general de la razón, que incluya en particular una reflexión y una acción morales, sea erosionada por la expansión de los modos técnicos de acción y de juicio. Dado que los afectados no pueden preservar plenamente el "antiguo" significado de sus palabras al margen de un desarrollo (general) semejante, el significado de las palabras se ve afectado por un proceso de esa índole. Puede alterarse o desaparecer, y esto es entonces un fenómeno "público". A fin de cuentas, prácticamente nadie puede conocer el antiguo arraigo de las palabras en nuestras vidas (las antiguas formas de entender la vida). De esta manera, podríamos en particular llegar al punto en que no hubiese una base para un argumento trascendental, en la medida en que las reconstrucciones científicas y técnicas socavaran desde el interior, por así decir, la semántica actual del mundo vital.

Podemos considerar una decadencia de la cultura de la razón -no sólo la definitoria "división de la racionalidad" - como una (deplorable) perspectiva de nuestra época, e incorporarnos a los esfuerzos por mantener una cultura racional con juicios y prácticas morales. Sin embargo, debemos tener cuidado en no reconstruir toda la gramática de la razón práctica dentro de un marco tomado de una determinada idea (o determinadas versiones) de la argumentación moral. El razonamiento moral no es el fundamento ni la forma de la racionalidad práctica en su totalidad.

Si con estas indicaciones estoy en lo correcto - y estoy bastante seguro de estar en lo correcto-, entonces carecen de sentido las pretensiones gramaticales de exclusividad de las reconstrucciones conceptuales del concepto de razón que se hallan en conflicto, las cuales marcan con su sello el desarrollo de la ética filosófica, de la teoría del bienestar público y la teoría de la decisión, pero también determinan a menudo el análisis de la racionalidad científica. Y esto significa que para nosotros las disputas filosóficas que se llevan aquí a cabo están extraviadas por la forma que asumen. Surgen de un malentendido gramatical sobre la razón: la gramática de una cultura adquiere sin más - sin que en casos semejantes existan circunstancias conceptuales necesarias - la forma de un principio (universalmente aplicable).

¿No tiene algo afirmativo la indicación sobre la práctica multifacética del juzgar, que está vinculada con las pretensiones de racionalidad? ¿No necesitamos los principios y criterios (generales) 
de la razón para conservar el sentido crítico del uso de la razón y de su gramática?

Pero lo que llamé cultura de la razón tiene que ver con una práctica del juzgar bien determinada. La cultura de la razón es, podríamos decir, normativa: esta es precisamente una característica de su gramática.

También las reglas dé un juego nos sirven para juzgar como correcta o incorrecta la práctica del juego; nos sirven como "norma" del jugar correcto, y, si nos fijamos bien, en este caso las reglas no tienen ningún principio que reúna (caracterice) todas sus aplicaciones correctas. La normatividad de la cultura de la razón no depende, como vemos; de nuestros prejuicios sobre la teoría de la definición en cuanto a un trato preciso (claro) con la palabra "razón" y su contexto lingüístico. Por eso la normatividad no nos abandona cuando luchamos contra estos prejuicios. Por el contrario, cuando ya no manejamos construcciones deductivas y conceptuales insostenibles, reforzamos un uso crítico de la razón suficientemente extendido. Pues aunque estos esfuerzos siempre fracasen o sólo alcancen una plausibilidad parcial, allanan a fin de cuentas el camino para el trato pluralista o ideológico-particular con las pretensiones de racionalidad.

Por otra parte, constatamos directamente cómo está configurado nuestro uso de las dictaminantes palabras "razonable" o "racional". Lo que nos interesa son los hechos gramaticales de esos usos en contraste con las falsas representaciones que nos hacemos de esos hechos. Y aprobamos los hechos que constatamos mediante una invitación a atenerse a ellos. ¿Acaso no es ésta una actitud descriptiva, y luego afirmativa, que impide la crítica necesaria de nuestro uso del lenguaje?- Pero esto es válido también para los principios de la razón. También éstos deben por lo regular acertar en el núcleo justificable del hecho cultural de la "razón", pues de otro modo caeríamos en la situación de querer traer al mundo algo que todavía no existe en absoluto en él. También un principio de razón se asocia entonces con la mera invitación: “iVe lo que hago y cómo hablo! ¡Únete!”

¿Es por tanto la afirmación el destino de la autorreflexión filosófica? En cuanto al uso de la razón, ¿tenemos que dejar todo tal como está? ¿No podría alguien referirse al llamado mal uso o uso unilateral (fáctico) de la razón y decirnos que éste es el significado más correcto, el mejor, el esencial de las palabras correspondientes? ¿Y cómo decidimos entonces - sin criterios que en cierto modo se encuentren al margen del conflicto, al margen del uso fáctico?

Si la práctica racional, en particular la argumentación racional, 
no nos proporciona por sí misma los medios de su juicio interno, de su limitación y su desarrollo, ¿cómo los obtendremos? ¿No es absurdo sostener que alguien pudiera inventar de manera privada la cultura de la razón y después difundirla? Eso significa que debemos tener los pies sobre el suelo de la razón si queremos movernos en ella y con ella. Reconocemos por eso que nunca podremos prescindir de los hechos gramaticales del uso de la razón.

Me parece importante una segunda aclaración: que no podamos reducir definitoriamente la cultura de la razón a un principio universal de delimitación (comprenderla sobre la base de éste), no debe confundirse con un argumento contra los rasgos universales, ante todo en los elementos morales de la razón (ni mucho menos contra el carácter formal de una gramática de la racionalidad). También aquí mis reflexiones solamente deben y pueden apuntar a ver lo formal y lo universal en una cultura de la razón desde la perspectiva gramatical correcta.

La universalidad de la razón práctica se opone, en cuanto a los propósitos terminológicos que son habituales en este contexto, a las llamadas orientaciones y realizaciones vitales particulares. Y la palabra "particular" puede aquí tener dos significados:

Por un lado, excluimos con ella lo que está fuera de nuestro (contexto de) vida común o lo que se ubica en el nivel del entendimiento, del análisis lingüístico, fuera de la "generalidad" de nuestras instituciones prácticas y lingüísticas. Queremos entonces decir que las palabras y las prácticas de las pretensiones de razón, nos rodean como una parte de esta generalidad, no son cosa de la mera concepción (subjetiva) y de la decisión de individuos, grupos, partidos, etcétera.

Por otra parte, el reproche de particularidad debe concernir a la gramática misma de la pretensión de razón y no (solamente) al hecho de que ésta no se disgregue en culturas particularés privadas de nuestra vida, en morales particulares, que no represente una cuestión de gusto o de interés. Aquí, en este segundo caso, la generalidad en el primer sentido ya está supuesta y se trata del contenido conceptual y práctico para el cual vale. Sobre este "contenido", a saber, la forma gramatical (general) de lo racional práctico, se discute con las palabras "particularidad" y (versus) "universalidad".

Si queremos explicarle a alguien lo universal de la cultura gramatical de la razón, ¿qué es lo que debemos hacer? ¿A qué nos podemos remitir? Aquí evidentemente hay que considerar muchas cosas, la mayor parte de las cuales fueron ya presentadas cuando pasamos revista a esta gramática: 
-que en situaciones determinadas nos apoyamos (en diferentes respectos) en orientaciones formuladas (y dirigidas) en forma general; -que la ponderación racional no reconoce gramaticalmente ninguna posición privilegiada ni en el nivel de la participación ni en el nivel de los argumentos;

-que reconocemos los intereses de los demás, consideramos la inclusión de todos los afectados como una perspectiva de nuestra ponderación;

-que abandonamos la perspectiva limitada de nuestras propias situaciones vitales y de nuestros intereses en favor de la perspectiva de una reflexión imparcial;

-que nos orientamos poniendo la mira en una comunidad humana libremente compartida;

-en relación con ello, también hacemos el reconocimiento moral reć́proco de la otra persona.

¿Existe aquí un criterio (ahora el de la universalidad) que satisfagán (deban satisfacer) todos estos puntos de vista y otros más? ¿No es más bien que estos puntos de vista (y otros más) - pero nada más allá de ellos- explican en qué múltiple sentido la cultura de nuestra razón incluye formas gramaticales de la superación de una práctica de orientación particular (individual, subjetiva)? Estas formas pertenecen, por así decir, a la fisonomía gramatical de la razón práctica, que en este sentido posee rasgos de lo universal, de lo imparcial, de lo transubjetivo.

Entonces también las palabras "formal" y "universal" nos engañan aparentando que podríamos operar aquí con elementos de una definición o de una construcción terminológica. Que debemos cuidarnos de manejar aquí esto, èn vez de una fenomenología múltiple, lo muestra también la enorme y característica opacidad, o el contenido tautológico, que se adhiere a las fórmulas que se pretenden definitorias. Además parecería que pueden funcionar en derivaciones analíticas o presuposicionales. Entre determinadas reglas del discurso y los principios de universalidád no se da sin embargo una relación de derivación del uno a partir del otro (como se sostiene, por ejemplo, en las ya mencionadas notas de Habermas para un programa de fundamentación de la ética del discurso), sino más bien la relación de la exposición gramatical de un contexto (de la cultura de razón) desde diferentes lados, de un contexto al que pertenecen tanto las reglas del discurso como los principios de universalidad.

Más allá de nuestra experiencia con los fenómenos e instituciones posibles, no sabemos lo que significan precisamente, en una consi- 
deración estricta (no exacta): simetría práctica de las posiciones discursivas, consideración de todos los afectados, superación de la (propia) subjetividad, imparcialidad, reconocimiento del otro y varias cosas más. Cada una de estas expresiones, decimos, no adquiere un perfil semántico más claro más que mediante su inserción en una plétora de experiencias, prácticas, reglamentaciones y actitudes. $Y$ todo esto está unido gramaticalmente en una complicada red, fuera de los límites de las formulaciones adecuadas en cada caso. Esta red, y no proposiciones o descripciones que contienen significativamente en un determinado lugar las palabras "general" o "forma", es lo que exhibe el discurso de la universalidad de la razón.

¿Deberíamos entonces mantenernos alejados del todo de definiciones o de reconstrucciones de lo racional? Tal vez para su sorpresa, diré que no, si prestamos atención a su lugar gramatical, a su limitado papel conceptual. Las diferentes definiciones de lo racional funcionan en el interior de la cultura de la razón; si son adecuadas "localmente", pertenecen a la red gramatical de esta cultura. $\mathrm{Y}$ no debemos entenderlas como competitivas determinaciones filosóficas de sus límites (de los límites gramaticales de una cultura de la razón). 